\title{
High-energy reconstruction for single and double cascades using the KM3NeT detector
}

\section{Thijs van Eeden, ${ }^{a, *}$ Jordan Seneca $^{a, *}$ and Aart Heijboer ${ }^{a}$ on behalf of the KM3NeT \\ Collaboration}

(a complete list of authors can be found at the end of the proceedings)

${ }^{a}$ Nikhef,

Science Park 105, 1098 XG Amsterdam, Netherlands

E-mail: tjuanve@nikhef.nl, jseneca@nikhef.nl

The discovery of a high-energy cosmic neutrino flux has paved the way for the field of neutrino astronomy. For a large part of the flux, the sources remain unidentified. The KM3NeT detector, which is under construction in the Mediterranean sea, is designed to determine their origin. KM3NeT will instrument a cubic kilometre of seawater with photomultiplier tubes that detect Cherenkov radiation from neutrino interaction products with nanosecond precision. For single cascade event signatures, KM3NeT already showed that it can reach degree-level resolutions, greatly increasing the use of these neutrinos for astronomy. In this contribution, we further refine the cascade reconstruction by making a more detailed model of the neutrinos events and including additional information on the hit times. The arrival time of light can be used to improve the identification of double cascade signatures from tau neutrinos, and the angular resolution of both single and double cascade signatures. Sub-degree resolution is achieved in both cases.

37th International Cosmic Ray Conference (ICRC 2021)

July 12th - 23rd, 2021

Online - Berlin, Germany

\footnotetext{
*Presenter
} 


\section{Introduction}

\subsection{Neutrino astronomy}

Neutrino properties make it an excellent complement to other cosmic messengers. The neutrality of the neutrino prevents deflections due to ambient magnetic fields when travelling to the Earth. Further, very low cross-sections enable the study of particle accelerators closer to the source, as well as preventing attenuation of the neutrino flux on the way to the Earth. The probing power of the neutrino as a cosmic messenger and implications for astroparticle physics and the understanding of cosmic ray production mechanisms and sources has been extensively documented [1,2].

A cosmic neutrino flux has been discovered by the IceCube detector [3], and while observations hinting at neutrino origins have been made [4], the sources of cosmic neutrinos remain elusive.

To enable the discovery of neutrino sources, a good angular resolution of the detector is crucial. For this reason, the track signatures caused by $v_{\mu}$ charged current interactions have been of the highest interest for searching for neutrino sources, IceCube achieving a resolution of $0.25^{\circ}$ [5], and below $0.1^{\circ}$ for KM3NeT/ARCA [6]. However, about two thirds of cosmic neutrinos are expected to produce a signature that is not a track, while currently only $20 \%$ of observed IceCube events have been tracks [7]. A good angular resolution on cascade events therefore represents an encouraging opportunity for neutrino source discovery. For cascades, IceCube achieves a resolution of $10^{\circ}$ at best [8], while the current high energy KM3NeT cascade reconstruction algorithm achieves a resolution of $1.5^{\circ}$ at best [9]. This work aims at improving further on the cascade signature reconstruction, and implementing the reconstruction for the most common $v_{\tau}$ charged current signature.

\subsection{KM3NeT/ARCA}

$\mathrm{KM} 3 \mathrm{NeT}$ is two neutrino detectors currently under construction on the bottom of the Mediterranean sea. ORCA is the component focusing on oscillation studies of atmospheric neutrinos travelling through the Earth. ARCA is the high energy neutrino telescope complement and subject of this work, located in Portopalo di Capo Passero in Sicily, Italy. The detector consists of a 3-D grid of digital optical modules (DOMs) that each contain 31 photomultiplier tubes (PMTs) [10]. The modules are mounted to vertical detection units (DUs) containing 18 DOMs spaced about 38 meters apart. The DUs are mounted to the seafloor and grouped in building blocks of 115 DUs with an average horizontal spacing of 95 meters. The complete ARCA detector will consist of two building blocks [9].

\subsection{Direction reconstruction of cascades}

The two main neutrino event signatures detected by KM3NeT are tracks and cascades. Hadronic cascades are produced at the vertex of all charged-current (CC) and neutral-current (NC) neutrino interactions. At high energies, the neutrino interacts through deep inelastic scattering, kicking out an energetic quark which forms the hadronic cascade. For CC interactions, an additional lepton corresponding to the neutrino flavour is produced. For electrons, this leads to an electromagnetic cascade, and thus a cascade signature, while muons travel through large parts of the detector. Highly-relativistic muons can travel several kilometers through seawater while producing Cherenkov light resulting in an extended track signature when comparing with other events. Muons 
produced by cosmic rays interacting in the atmosphere also reach and travel through the detector. The tau has a mean lifetime of $2.903 \pm 0.005 \times 10^{-13} \mathrm{~s}$ and decays into hadrons and leptons [11]. The branching ratio to an electron or hadrons is 0.8261 which can result in a double cascade signature for high enough $\tau$-decay lengths. The cascades are separated by an average $5 \frac{\mathrm{cm}}{\mathrm{TeV}}$ due to time dilatation. The double cascade signature distinguishes itself from regular cascades when the tau length is more than a few meters, which is the typical particle cascade size at TeV energies.

The direction reconstruction of the track signature utilizes the detection time of Cherenkov light on PMTs near the track in order to fit the direction of the muon. The standard KM3NeT cascade reconstruction does not use the detection times to reconstruct the direction, but uses the presence or absence of hits on each PMT as a measure of the light intensity. This work presents two new reconstruction algorithms for single and double cascades where we include the timing information for direction reconstruction and improve on the modelling of cascades by taking into account their elongated shape. This effort is motivated by the idea that an improved description of the events and additional information leads to an improvement in reconstruction performance. The improved model with timing information is also expected to improve due to the lever arm effect which the track reconstruction also benefits from. The early and late parts of both single and double cascade events are strongly restricted in position thanks to the arrival time of the light that they produce. This places the start and end of the event along the direction of the event, achieving a better angular resolution. The method is described first in 2 , followed by the performance for single and double cascades in 3 . We conclude with a summary and short outlook in 4.

\section{Method}

The standard KM3NeT single cascade reconstruction (Aashowerfit) fits the spatial Cherenkov profile to the PMT and hit positions to estimate the direction and energy of a neutrino. The vertex and time of the event are estimated by minimizing the hit time residuals assuming isotropic light emission from the shower maximum.

The likelihood function is built from the Poisson probability of no hits occurring. If a hit did occur on the PMT, the complementary probability is used. The likelihood is then the product of the Poisson probabilities for each PMT hit or no-hit status,

$$
\mathcal{L}_{\text {hit/no-hit }}=\prod_{\text {hit PMTs }} 1-e^{-N_{s}-N_{b g}} \prod_{\text {no-hit PMTs }} e^{-N_{s}-N_{b g}}
$$

where $N_{s}$ is the expected number of photo-electrons (n.p.e.) due to a cascade hypothesis and $N_{b g}$ is the expected background n.p.e. $N_{b g}$ is obtained by fitting ARCA data to a constant ${ }^{40} \mathrm{~K}$ background hypothesis. For Aashowerfit, $N_{s}$ is obtained from a model built from interpolated Monte Carlo simulations of $1 \mathrm{PeV} v_{e} \mathrm{CC}$ events. In the new reconstruction, $N_{s}$ is obtained from a semi-analytical Cherenkov light model built with interpolated simulations of electromagnetic cascade energy deposition and light emission profiles. Aashowerfit is used as a prefit for the single and double cascade reconstructions using timing information.

\subsection{First hit information}

The hit/no-hit likelihood from equation 1 is the sum of the probabilities for PMTs to have seen light or not during a cascade event. This likelihood can be extended with the information of when 
each hit PMT was hit. The analogue signals from the PMTs are digitized inside the DOMs and return a time, at which the signal surpasses a threshold, and a time-over-threshold (ToT). Photons on the same PMT that are closely spaced in time result in a single hit with the time of the first hit. Only the hit time of the first hit on every PMT is used when including timing information. Figure 1 shows the time residuals for a single cascade.

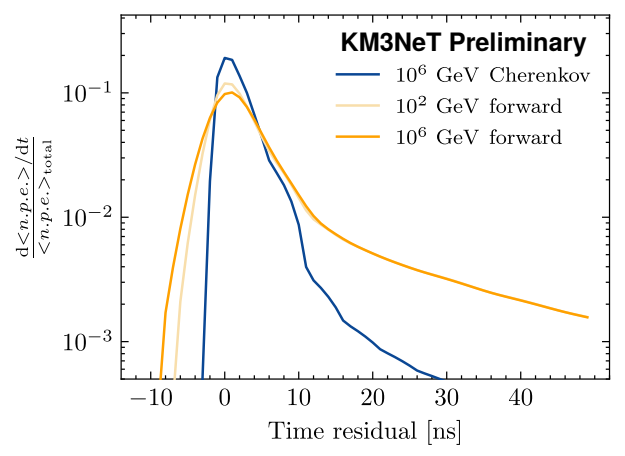

Figure 1: Time residuals for a $10^{2}$ and $10^{6} \mathrm{GeV}$ single cascade, for a PMT in the cherenkov cone, and in the forward direction. Note that different profiles are observed depending on where the event is pointing with respect to the PMT.

The hit time information is crucial in the reconstruction of track-like events induced by muons. A highly-relativistic muon travels long distances under water while producing Cherenkov light. The Cherenkov wavefront is the first light hitting a PMT and this information is used to fit the direction of the muon, also benefiting from the lever-arm effect. For cascades, Aashowerfit utilizes the timing information to fit the vertex position and time, but ignores the time in the direction reconstruction.

\subsection{Fit routine}

The likelihood can be re-defined to include a time component

$$
\mathcal{L}_{\text {time }}=\prod_{1 \text { st hits }} P_{1 \mathrm{st}}(t)
$$

where $P_{1 \text { st }}(t)$ is the probability density for the first hit to occur at time $t$ given that a hit occurs. The full likelihood can then be written as

$$
\mathcal{L}=\prod_{1 \text { st hits }} P_{1 \mathrm{st}}(t) \prod_{\text {hit PMTs }} 1-e^{-N_{s}-N_{b g}} \prod_{\text {no-hit PMTs }} e^{-N_{s}-N_{b g}}
$$

using both the hit/no-hit information and the timing information. The reconstruction is a maximum likelihood estimator of the likelihood in eq. 3.

\subsubsection{Single cascades}

The single cascade algorithm using timing information consists of three steps:

1. Aashowerfit prefit.

2. Precision position and time prefit. 


\section{Full likelihood maximisation using single cascade hypothesis.}

The timing information of the first hits is sensitive to the position and time of vertex of the cascade. A good vertex position and time prefit is therefore constructed using the timing of the first hits only. The Aashowerfit vertex time is used as a starting point for the prefit. For the position, 13 positions are chosen. The Aashowerfit vertex position, and 12 positions equally spaced on a sphere of radius $1 \mathrm{~m}$, centered on the Aashowerfit vertex position. Starting from each position, the first hits only are used to reconstruct the position and time of the vertex, using the first hit likelihood and elongated model. Out of the 13 results, the track with the highest likelihood is picked as the prefit result. This process is re-iterated once with the new best-fit vertex to further improve the prefit and kick outliers out of local minima. The full likelihood, eq. 3, is then maximized with the non-hit PMTs, and the time of the hit PMTs.

\subsubsection{Double cascades}

The double cascade algorithm consists of three steps:

1. Aashowerfit prefit

2. Tau length prefit

3. Time likelihood maximisation using double cascade hypothesis.

The Aashowerfit prefit assumes a single cascade and estimates a vertex somewhere in between or in front of the first and second cascade. The tau length prefit searches along the Aashowerfit direction to find the position of the first and second cascade. This results in a first estimate of the travelling length of the tau lepton. The results of the prefits are used as starting values in the final fit where the time likelihood (equation 2) is maximised with respect to the vertex of the first cascade, the direction of both cascades, the length of the tau and the energy division of the first and second cascade. The high-energy cascades are assumed to be colinear and separated by the speed of light. 


\section{Performance}

\subsection{Single cascades}

Figure 2 shows the median angular deviation and spread of relative energy difference for the single cascade reconstruction. There is a clear improvement in the median when including timing information. The spread of relative energy difference between reconstructed energy and true energy also shows an improvement.

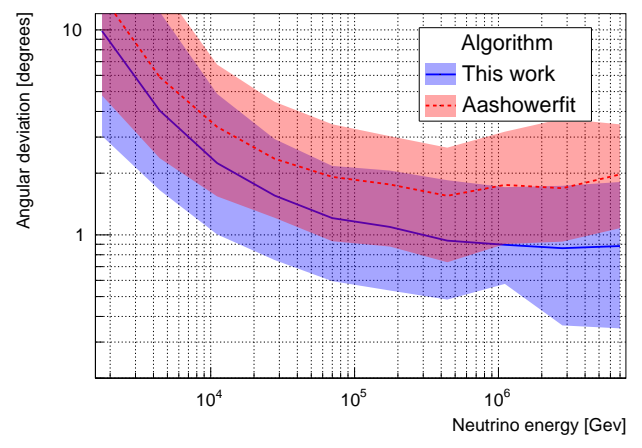

(a)

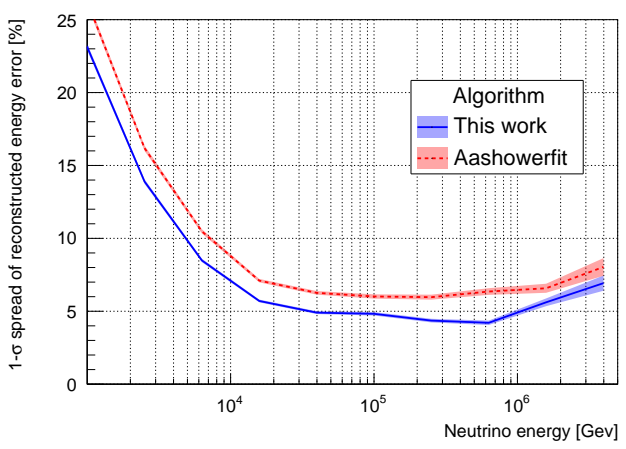

(b)

Figure 2: Direction (a) and energy (b) reconstruction performance for single cascade reconstruction.

Figure 3 shows the position deviation for the single cascade reconstruction. The position resolution improves when including timing information in the elongated cascade model for both the transverse and longitudinal plane.

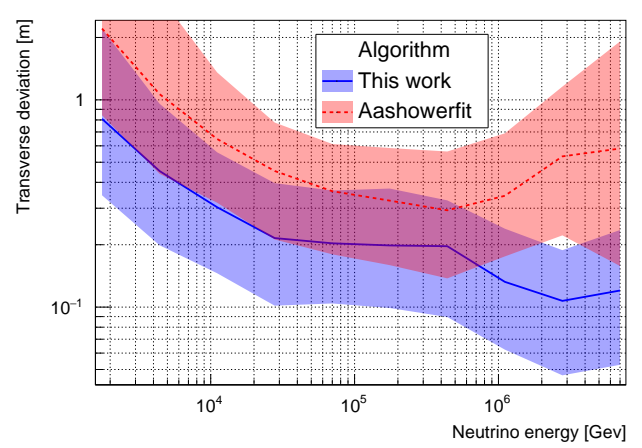

(a)

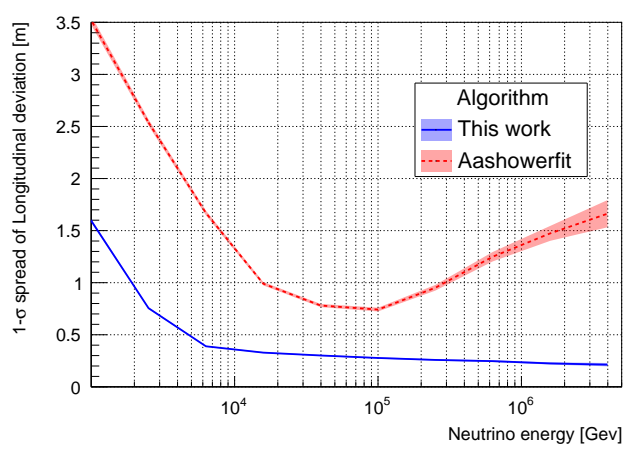

(b)

Figure 3: Transverse (a) and longitudinal (b) position deviation for single cascade reconstruction. 


\subsection{Double cascades}

Figure 4 shows the angular deviation between the reconstructed direction and true direction for double cascade events. The events are weighted with a $1.2 \cdot 10^{-8} \cdot E^{-2} \mathrm{GeV}^{-1} \mathrm{sr}^{-1} \mathrm{~s}^{-1} \mathrm{~cm}^{-2}$ spectrum and there has been an event selection based on the Aashowerfit output. The reconstructed vertex is required to be within the inner half volume of the detector and the reconstructed energy above $100 \mathrm{TeV}$.

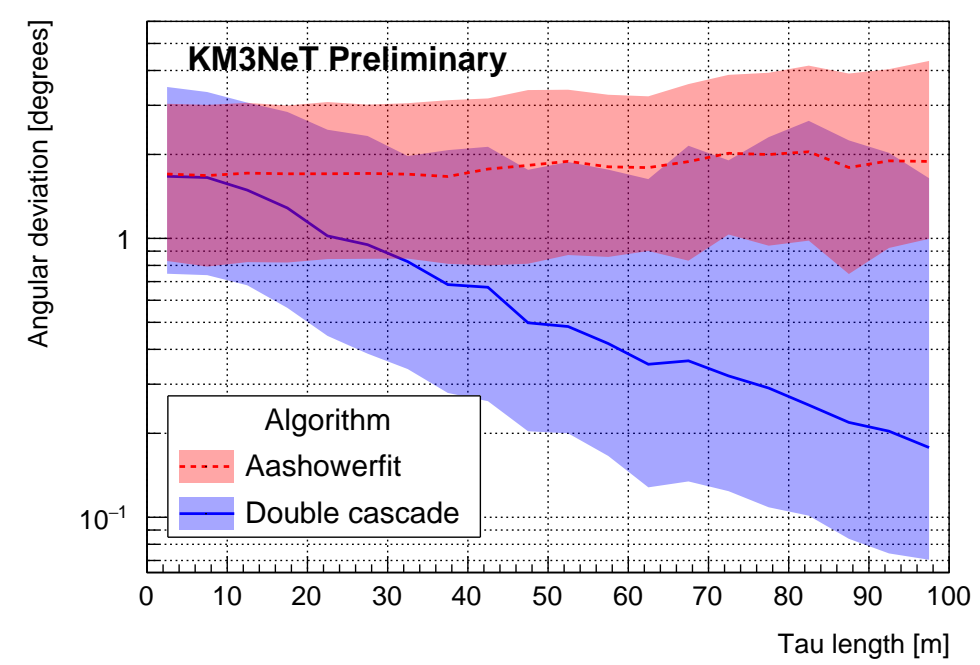

Figure 4: Angular deviation as a function of the true tau length.

Figure 5 shows the reconstructed length error and the reconstructed visible energy error.

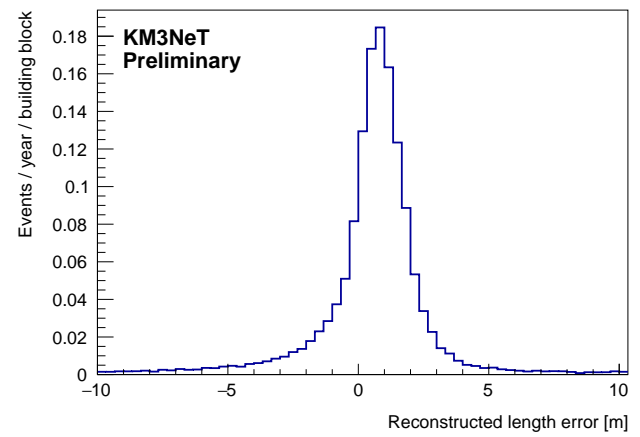

(a)

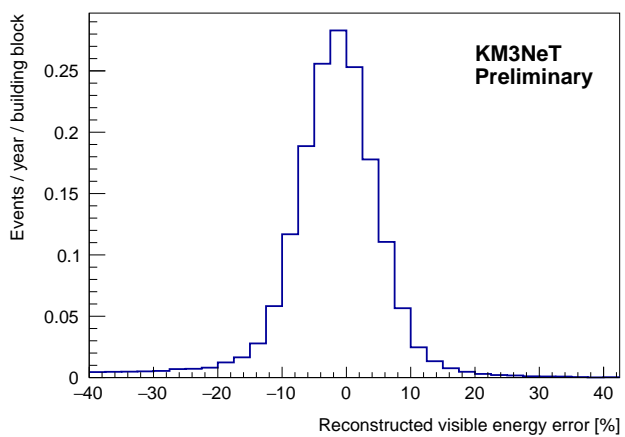

(b)

Figure 5: Reconstructed length (a) and energy error (b) for the double cascade reconstruction. The median and $68 \%$ quantiles of the reconstructed length error are $0.72_{-1.95}^{+1.23}$ meters. The median and $68 \%$ quantiles of the reconstructed energy error are $-1.75_{-6.90}^{+6.11} \%$. 


\section{Summary}

We have a presented two new reconstruction algorithms which use the timing information and elongation emission profile of cascades. The median angular deviation improves over the whole energy range and drops below 1 degree for single cascades of $300 \mathrm{TeV}$. We also see an improvement in the position resolution that benefits the double cascade reconstruction. For double cascades, the angular deviation drops below 1 degree for tau lengths longer than 25 meters. The improvement with respect to single cascades comes from a stronger lever-arm effect due to the large extension of double cascade events. We find a reconstructed length error spread of 3.17 meters and a reconstructed visible energy error spread of $13 \%$.

\section{References}

[1] Becker, Julia K. "High-energy neutrinos in the context of multimessenger astrophysics." Physics Reports 458.4-5 (2008): 173-246.

[2] Learned, John G., and Karl Mannheim. "High-energy neutrino astrophysics." Annual Review of Nuclear and Particle Science 50.1 (2000): 679-749.

[3] Aartsen, M. G., et al. "Observation and Characterization of a Cosmic Muon Neutrino Flux from the Northern Hemisphere using six years of IceCube data." The Astrophysical Journal 833.1 (2016): 3 .

[4] Telescope, Liverpool, et al. "Multimessenger observations of a flaring blazar coincident with high-energy neutrino IceCube-170922A." Science 361.6398 (2018).

[5] Aartsen, M. G., et al. "Time-integrated neutrino source searches with 10 years of IceCube data." Physical review letters 124.5 (2020): 051103.

[6] Melis, K., et al. "KM3NeT/ARCA Event Reconstruction Algorithms." Proceedings, 35th International Cosmic Ray Conference (ICRC2017). 2017.

[7] Kopper C, Giang W and Kurahashi N(for the IceCube Collaboration) Proc. 34th Int.Cosmic Ray Conf (2015): vol 001 p 1081 PoS

[8] Aartsen, M. G., et al. "Search for astrophysical sources of neutrinos using cascade events in IceCube." The Astrophysical Journal 846.2 (2017): 136.

[9] Adrian-Martinez, Silvia, et al. "Letter of intent for KM3NeT 2.0." Journal of Physics G: Nuclear and Particle Physics 43.8 (2016): 084001.

[10] R. Bruijn, "The KM3NeT Digital Optical Module and Detection Unit." (2019).

[11] C. Tanabashi et al., "Particle Data Group", Phys. Rev. D 98, 030001 (2018) 620-624.

[12] C. Kopper, "Performance studies for the KM3NeT neutrino telescope." (2012). 


\section{Full Author List: KM3NeT Collaboration}

M. Ageron ${ }^{1}$, S. Aiello ${ }^{2}$, A. Albert ${ }^{3,55}$, M. Alshamsi ${ }^{4}$, S. Alves Garre ${ }^{5}$,Z. Aly ${ }^{1}$, A. Ambrosone ${ }^{6,7}$, F. Ameli ${ }^{8}$, M. Andre ${ }^{9}$, G. Androulakis ${ }^{10}$, M. Anghinolfi ${ }^{11}$, M. Anguita ${ }^{12}$, G. Anton ${ }^{13}$, M. Ardid $^{14}$, S. Ardid $^{14}$, W. Assal ${ }^{1}$, J. Aublin ${ }^{4}$, C. Bagatelas ${ }^{10}$, B. Baret ${ }^{4}$, S. Basegmez du Pree ${ }^{15}$, M. Bendahman ${ }^{4,16}$, F. Benfenati ${ }^{17,18}$, E. Berbee ${ }^{15}$, A. M. van den Berg ${ }^{19}$, V. Bertin ${ }^{1}$, S. Beurthey ${ }^{1}$, V. van Beveren ${ }^{15}$, S. Biagi ${ }^{20}$, M. Billault ${ }^{1}$, M. Bissinger ${ }^{13}$, M. Boettcher ${ }^{21}$, M. Bou Cabo ${ }^{22}$, J. Boumaaza ${ }^{16}$, M. Bouta ${ }^{23}$, C. Boutonnet $^{4}$, G. Bouvet ${ }^{24}$, M. Bouwhuis ${ }^{15}$, C. Bozza ${ }^{25}$, H.Brânzaş ${ }^{26}$, R. Bruijn ${ }^{15,27}$, J. Brunner ${ }^{1}$, R. Bruno ${ }^{2}$, E. Buis ${ }^{28}$, R. Buompane ${ }^{6,29}$, J. Busto ${ }^{1}$, B. Caiffi ${ }^{11}$, L. Caillat ${ }^{1}$, D. Calvo ${ }^{5}$, S. Campion ${ }^{30,8}$, A. Capone ${ }^{30,8}$, H. Carduner ${ }^{24}$, V. Carretero ${ }^{5}$, P. Castaldi ${ }^{17,31}$, S. Celli ${ }^{30,8}$, R. Cereseto ${ }^{11}$, M. Chabab ${ }^{32}$, C. Champion ${ }^{4}$, N. $\mathrm{Chau}^{4}$, A. Chen ${ }^{33}$, S. Cherubinini ${ }^{20,34}$, V. Chiarella ${ }^{35}$, T. Chiarusi ${ }^{17}$, M. Circella ${ }^{36}$, R. Cocimano ${ }^{20}$, J. A. B. Coelho ${ }^{4}$, A. Coleiro ${ }^{4}$, M. Colomer Molla ${ }^{4,5}$, S. Colonges ${ }^{4}$, R. Coniglione ${ }^{20}$, A. Cosquer ${ }^{1}$, P. Coyle ${ }^{1}$, M. Cresta ${ }^{11}$, A. Creusot ${ }^{4}$, A. $\mathrm{Cruz}^{37}$, G. Cuttone ${ }^{20}$, A. D’Amico ${ }^{15}$, R. Dallier ${ }^{24}$, B. De Martino ${ }^{1}$, M. De Palma ${ }^{36,38}$, I. Di Palma ${ }^{30,8}$, A. F. Díaz ${ }^{12}$, D. DiegoTortosa $^{14}$, C. Distefano ${ }^{20}$, A. Domi ${ }^{15,27}$, C. Donzaud ${ }^{4}$, D. Dornic ${ }^{1}$, M. Dörr ${ }^{39}$, D. Drouhin ${ }^{3,55}$, T. Eberl ${ }^{13}$, A. Eddyamoui ${ }^{16}$, T. van Eeden ${ }^{15}$, D. van Eijk ${ }^{15}$, I. El Bojaddaini ${ }^{23}$, H. Eljarrari ${ }^{16}$, D. Elsaesser ${ }^{39}$, A. Enzenhöfer ${ }^{1}$, V. Espinosa ${ }^{14}$, P. Fermani ${ }^{30,8}$, G. Ferrara ${ }^{20,34}$, M. D. Filipović ${ }^{40}$, F. Filippini ${ }^{17,18}$, J. Fransen ${ }^{15}$, L. A. Fusco ${ }^{1}$, D. Gajanana ${ }^{15}$, T. Gal ${ }^{13}$, J. García Méndez ${ }^{14}$, A. Garcia Soto $^{5}$, E. Garçon ${ }^{1}$, F. Garufi ${ }^{6,7}$, C. Gatius ${ }^{15}$, N. Geißelbrecht ${ }^{13}$, L. Gialanella ${ }^{6,29}$, E. Giorgio ${ }^{20}$, S. R. Gozzini ${ }^{5}$, R. Gracia ${ }^{15}$, K. Graf ${ }^{13}$, G. Grella ${ }^{41}$, D. Guderian ${ }^{56}$, C. Guidi ${ }^{11,42}$, B. Guillon ${ }^{43}$, M. Gutiérrez ${ }^{44}$, J. Haefner ${ }^{13}$, S. Hallmann ${ }^{13}$, H. Hamdaoui ${ }^{16}$, H. van Haren ${ }^{45}$, A. Heijboer ${ }^{15}$, A. Hekalo ${ }^{39}$, L. Hennig ${ }^{13}$, S. Henry ${ }^{1}$, J. J. Hernández-Rey ${ }^{5}$, J. Hofestädt ${ }^{13}$, F. Huang ${ }^{1}$, W. Idrissi Ibnsalih ${ }^{6,29}$, A. Ilioni ${ }^{4}$, G. Illuminati ${ }^{17,18,4}$, C. W. James ${ }^{37}$, D. Janezashvili ${ }^{46}$, P. Jansweijer ${ }^{15}$, M. de Jong ${ }^{15,47}$, P. de Jong ${ }^{15,27}$, B. J. Jung ${ }^{15}$, M. Kadler ${ }^{39}$, P. Kalaczyński ${ }^{48}$, O. Kalekin ${ }^{13}$, U. F. Katz ${ }^{13}$, F. Kayzel $^{15}$, P. Keller ${ }^{1}$, N. R. Khan Chowdhury ${ }^{5}$, G. Kistauri ${ }^{46}$, F. van der Knaap ${ }^{28}$, P. Kooijman ${ }^{27,57}$, A. Kouchner ${ }^{4,49}$, M. Kreter ${ }^{21}$, V. Kulikovskiy ${ }^{11}$, M. Labalme ${ }^{43}$, P. Lagier ${ }^{1}$, R. Lahmann ${ }^{13}$, P. Lamare ${ }^{1}$, M. Lamoureux $^{14}$, G. Larosa ${ }^{20}$, C. Lastoria ${ }^{1}$, J. Laurence ${ }^{1}$, A. Lazo $^{5}$, R. Le Breton ${ }^{4}$, E. Le Guirriec ${ }^{1}$, S. Le Stum ${ }^{1}$, G. Lehaut ${ }^{43}$, O. Leonardi ${ }^{20}$, F. Leone ${ }^{20,34}$, E. Leonora ${ }^{2}$, C. Lerouvillois ${ }^{1}$, J. Lesrel ${ }^{4}$, N. Lessing ${ }^{13}$, G. Levi ${ }^{17,18}$, M. Lincetto ${ }^{1}$, M. Lindsey Clark ${ }^{4}$, T. Lipreau ${ }^{24}$, C. LLorens Alvarez ${ }^{14}$, A. Lonardo ${ }^{8}$, F. Longhitano ${ }^{2}$, D. Lopez-Coto ${ }^{44}$, N. Lumb ${ }^{1}$, L. Maderer ${ }^{4}$, J. Majumdar ${ }^{15}$, J. Mańczak ${ }^{5}$, A. Margiotta ${ }^{17,18}$, A. Marinelli ${ }^{6}$, A. Marini ${ }^{1}$, C. Markou $^{10}$, L. Martin ${ }^{24}$, J. A. Martínez-Mora ${ }^{14}$, A. Martini ${ }^{35}$, F. Marzaioli ${ }^{6,29}$,

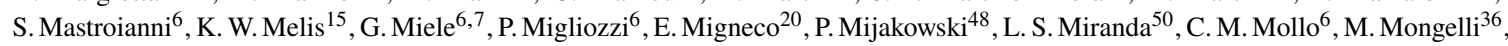
A. Moussa ${ }^{23}$, R. Muller ${ }^{15}$, P. Musico ${ }^{11}$, M. Musumeci ${ }^{20}$, L. Nauta ${ }^{15}$, S. Navas ${ }^{44}$, C. A. Nicolau ${ }^{8}$, B. Nkosi ${ }^{33}$, B. Ó Fearraigh ${ }^{15,27}$, M. O'Sullivan ${ }^{37}$, A. Orlando ${ }^{20}$, G. Ottonello ${ }^{11}$, S. Ottonello ${ }^{11}$, J. Palacios González ${ }^{5}$, G. Papalashviliite, R. Papaleo ${ }^{20}$, C. Pastore ${ }^{36}$, A. M. Păun ${ }^{26}$, G.E. Păvălaşş, G. Pellegrinini ${ }^{17}$, C. Pellegrino ${ }^{18,58}$, M. Perrin-Terrinin ${ }^{1}$, V. Pestel ${ }^{15}$, P. Piattelli ${ }^{20}$, C. Pieterse ${ }^{5}$, O. Pisanti ${ }^{6,7}$, C. Poirè ${ }^{14}$, V. Popa $^{26}$, T. Pradier $^{3}$, F. Pratolongo ${ }^{11}$, I. Probst ${ }^{13}$, G. Pühlhofer ${ }^{51}$, S. Pulvirenti ${ }^{20}$, G. Quéméner ${ }^{43}$, N. Randazzo ${ }^{2}$, A. Rapicavoli ${ }^{34}$, S. Razzaque $^{50}$, D. Real ${ }^{5}$, S. Reck ${ }^{13}$, G. Riccobene ${ }^{20}$, L. Rigalleau ${ }^{24}$, A. Romanov ${ }^{11,42}$, A. Rovelli ${ }^{20}$, J. Royon ${ }^{1}$, F. Salesa Greus ${ }^{5}$, D. F. E. Samtleben ${ }^{15,47}$, A. Sánchez Losa ${ }^{36,5}$, M. Sanguineti ${ }^{11,42}$, A. Santangelo ${ }^{51}$, D. Santonocito ${ }^{20}$, P. Sapienza ${ }^{20}$, J. Schmelling ${ }^{15}$, J. Schnabel ${ }^{13}$, M. F. Schneider ${ }^{13}$, J. Schumann ${ }^{13}$, H. M. Schutte ${ }^{21}$, J. Seneca ${ }^{15}$, I. Sgura ${ }^{36}$, R. Shanidze ${ }^{46}$, A. Sharma ${ }^{52}$, A. Sinopoulou ${ }^{10}$, B. Spisso ${ }^{41,6}$, M. Spurio ${ }^{17,18}$, D. Stavropoulos ${ }^{10}$, J. Steijger ${ }^{15}$, S. M. Stellacci ${ }^{41,6}$, M. Taiuti ${ }^{11,42}$, F. Tatone ${ }^{36}$, Y. Tayalati ${ }^{16}$, E. Tenllado ${ }^{44}$, D. Tézier ${ }^{1}$, T. Thakore ${ }^{5}$, S. Theraube ${ }^{1}$, H. Thiersen $^{21}$, P. Timmer $^{15}$, S. Tingay ${ }^{37}$, S. Tsagkli $^{10}$, V. Tsourapis ${ }^{10}$, E. Tzamariudaki ${ }^{10}$, D. Tzanetatos ${ }^{10}$, C. Valieri ${ }^{17}$, V. Van Elewyck ${ }^{4,49}$, G. Vasileiadis ${ }^{53}$, F. Versari ${ }^{17,18}$, S. Viola $^{20}$, D. Vivolo ${ }^{6,29}$, G. de Wasseige ${ }^{4}$, J. Wilms ${ }^{54}$, R. Wojaczyński ${ }^{48}$, E. de Wolf ${ }^{15,27}$, T. Yousfi ${ }^{23}$, S. Zavatarelli ${ }^{11}$, A. Zegarelli ${ }^{30,8}$, D. Zito ${ }^{20}$, J. D. Zornoza ${ }^{5}$, J. Zúñiga ${ }^{5}$, N. Zywucka ${ }^{21}$.

${ }^{1}$ Aix Marseille Univ, CNRS/IN2P3, CPPM, Marseille, France.

${ }^{2}$ INFN, Sezione di Catania, Via Santa Sofia 64, Catania, 95123 Italy.

${ }^{3}$ Université de Strasbourg, CNRS, IPHC UMR 7178, F-67000 Strasbourg, France.

${ }^{4}$ Université de Paris, CNRS, Astroparticule et Cosmologie, F-75013 Paris, France.

${ }^{5}$ IFIC - Instituto de Física Corpuscular (CSIC - Universitat de València), c/Catedrático José Beltrán, 2, 46980 Paterna, Valencia, Spain. ${ }^{6}$ INFN, Sezione di Napoli, Complesso Universitario di Monte S. Angelo, Via Cintia ed. G, Napoli, 80126 Italy.

${ }^{7}$ Università di Napoli “Federico II", Dip. Scienze Fisiche "E. Pancini”, Complesso Universitario di Monte S. Angelo, Via Cintia ed. G, Napoli, 80126 Italy.

${ }^{8}$ INFN, Sezione di Roma, Piazzale Aldo Moro 2, Roma, 00185 Italy.

${ }^{9}$ Universitat Politècnica de Catalunya, Laboratori d'Aplicacions Bioacústiques, Centre Tecnològic de Vilanova i la Geltrú, Avda. Rambla Exposició, s/n, Vilanova i la Geltrú, 08800 Spain.

${ }^{10}$ NCSR Demokritos, Institute of Nuclear and Particle Physics, Ag. Paraskevi Attikis, Athens, 15310 Greece.

${ }^{11}$ INFN, Sezione di Genova, Via Dodecaneso 33, Genova, 16146 Italy.

${ }^{12}$ University of Granada, Dept. of Computer Architecture and Technology/CITIC, 18071 Granada, Spain.

${ }^{13}$ Friedrich-Alexander-Universität Erlangen-Nürnberg, Erlangen Centre for Astroparticle Physics, Erwin-Rommel-Straße 1, 91058 Erlangen, Germany.

${ }^{14}$ Universitat Politècnica de València, Instituto de Investigación para la Gestión Integrada de las Zonas Costeras, C/ Paranimf, 1, Gandia, 46730 Spain.

${ }^{15}$ Nikhef, National Institute for Subatomic Physics, PO Box 41882, Amsterdam, 1009 DB Netherlands.

${ }^{16}$ University Mohammed V in Rabat, Faculty of Sciences, 4 av. Ibn Battouta, B.P. 1014, R.P. 10000 Rabat, Morocco.

${ }^{17}$ INFN, Sezione di Bologna, v.le C. Berti-Pichat, 6/2, Bologna, 40127 Italy.

\footnotetext{
${ }^{1}$ also at Dipartimento di Fisica, INFN Sezione di Padova and Università di Padova, I-35131, Padova, Italy
} 
${ }^{18}$ Università di Bologna, Dipartimento di Fisica e Astronomia, v.le C. Berti-Pichat, 6/2, Bologna, 40127 Italy.

${ }^{19}$ KVI-CART University of Groningen, Groningen, the Netherlands.

${ }^{20}$ INFN, Laboratori Nazionali del Sud, Via S. Sofia 62, Catania, 95123 Italy.

${ }^{21}$ North-West University, Centre for Space Research, Private Bag X6001, Potchefstroom, 2520 South Africa.

${ }^{22}$ Instituto Español de Oceanografía, Unidad Mixta IEO-UPV, C/ Paranimf, 1, Gandia, 46730 Spain.

${ }^{23}$ University Mohammed I, Faculty of Sciences, BV Mohammed VI, B.P. 717, R.P. 60000 Oujda, Morocco.

${ }^{24}$ Subatech, IMT Atlantique, IN2P3-CNRS, Université de Nantes, 4 rue Alfred Kastler - La Chantrerie, Nantes, BP 2072244307 France.

${ }^{25}$ Università di Salerno e INFN Gruppo Collegato di Salerno, Dipartimento di Matematica, Via Giovanni Paolo II 132, Fisciano, 84084 Italy.

${ }^{26}$ ISS, Atomistilor 409, Măgurele, RO-077125 Romania.

${ }^{27}$ University of Amsterdam, Institute of Physics/IHEF, PO Box 94216, Amsterdam, 1090 GE Netherlands.

${ }^{28}$ TNO, Technical Sciences, PO Box 155, Delft, 2600 AD Netherlands.

${ }^{29}$ Università degli Studi della Campania "Luigi Vanvitelli", Dipartimento di Matematica e Fisica, viale Lincoln 5, Caserta, 81100 Italy.

${ }^{30}$ Università La Sapienza, Dipartimento di Fisica, Piazzale Aldo Moro 2, Roma, 00185 Italy.

${ }^{31}$ Università di Bologna, Dipartimento di Ingegneria dell'Energia Elettrica e dell'Informazione "Guglielmo Marconi", Via dell'Università 50, Cesena, 47521 Italia.

${ }^{32}$ Cadi Ayyad University, Physics Department, Faculty of Science Semlalia, Av. My Abdellah, P.O.B. 2390, Marrakech, 40000 Morocco.

${ }^{33}$ University of the Witwatersrand, School of Physics, Private Bag 3, Johannesburg, Wits 2050 South Africa.

${ }^{34}$ Università di Catania, Dipartimento di Fisica e Astronomia "Ettore Majorana", Via Santa Sofia 64, Catania, 95123 Italy.

${ }^{35}$ INFN, LNF, Via Enrico Fermi, 40, Frascati, 00044 Italy.

${ }^{36}$ INFN, Sezione di Bari, via Orabona, 4, Bari, 70125 Italy.

${ }^{37}$ International Centre for Radio Astronomy Research, Curtin University, Bentley, WA 6102, Australia.

${ }^{38}$ University of Bari, Via Amendola 173, Bari, 70126 Italy.

${ }^{39}$ University Würzburg, Emil-Fischer-Straße 31, Würzburg, 97074 Germany.

${ }^{40}$ Western Sydney University, School of Computing, Engineering and Mathematics, Locked Bag 1797, Penrith, NSW 2751 Australia.

${ }^{41}$ Università di Salerno e INFN Gruppo Collegato di Salerno, Dipartimento di Fisica, Via Giovanni Paolo II 132, Fisciano, 84084 Italy.

${ }^{42}$ Università di Genova, Via Dodecaneso 33, Genova, 16146 Italy.

${ }^{43}$ Normandie Univ, ENSICAEN, UNICAEN, CNRS/IN2P3, LPC Caen, LPCCAEN, 6 boulevard Maréchal Juin, Caen, 14050 France.

${ }^{44}$ University of Granada, Dpto. de Física Teórica y del Cosmos \& C.A.F.P.E., 18071 Granada, Spain.

${ }^{45} \mathrm{NIOZ}$ (Royal Netherlands Institute for Sea Research), PO Box 59, Den Burg, Texel, 1790 AB, the Netherlands.

${ }^{46}$ Tbilisi State University, Department of Physics, 3, Chavchavadze Ave., Tbilisi, 0179 Georgia.

${ }^{47}$ Leiden University, Leiden Institute of Physics, PO Box 9504, Leiden, 2300 RA Netherlands.

${ }^{48}$ National Centre for Nuclear Research, 02-093 Warsaw, Poland.

${ }^{49}$ Institut Universitaire de France, 1 rue Descartes, Paris, 75005 France.

${ }^{50}$ University of Johannesburg, Department Physics, PO Box 524, Auckland Park, 2006 South Africa.

${ }^{51}$ Eberhard Karls Universität Tübingen, Institut für Astronomie und Astrophysik, Sand 1, Tübingen, 72076 Germany.

${ }^{52}$ Università di Pisa, Dipartimento di Fisica, Largo Bruno Pontecorvo 3, Pisa, 56127 Italy.

${ }^{53}$ Laboratoire Univers et Particules de Montpellier, Place Eugène Bataillon - CC 72, Montpellier Cédex 05, 34095 France.

${ }^{54}$ Friedrich-Alexander-Universität Erlangen-Nürnberg, Remeis Sternwarte, Sternwartstraße 7, 96049 Bamberg, Germany.

${ }^{55}$ Université de Haute Alsace, 68100 Mulhouse Cedex, France.

${ }^{56}$ University of Münster, Institut für Kernphysik, Wilhelm-Klemm-Str. 9, Münster, 48149 Germany.

${ }^{57}$ Utrecht University, Department of Physics and Astronomy, PO Box 80000, Utrecht, 3508 TA Netherlands.

${ }^{58}$ INFN, CNAF, v.le C. Berti-Pichat, 6/2, Bologna, 40127 Italy. 